\title{
Active sorting of orbital angular momentum states of light with a cascaded tunable resonator
}

\author{
Shibiao Wei $\mathbb{1}^{1,2,3}$, Stuart K. Earl $\mathbb{1}^{2,3}$, Jiao Lin ${ }^{1,3,4}$, Shan Shan Kou² and Xiao-Cong Yuan (1)
}

\begin{abstract}
The orbital angular momentum (OAM) of light has been shown to be useful in diverse fields ranging from astronomy and optical trapping to optical communications and data storage. However, one of the primary impediments preventing such applications from widespread adoption is the lack of a straightforward and dynamic method to sort incident OAM states without altering the states. Here, we report a technique that can dynamically filter individual OAM states and preserve the incident OAM states for subsequent processing. Although the working principle of this technique is based on resonance, the device operation is not limited to a particular wavelength. OAM states with different wavelengths can resonate in the resonator without any additional modulation other than changing the length of the cavity. Consequently, we are able to demonstrate a reconfigurable OAM sorter that is constructed by cascading such optical resonators. This approach does not require specially designed components and is readily amenable to integration into potential applications.
\end{abstract}

\section{Introduction}

The optical orbital angular momentum (OAM) carried by vortex beams has been shown to be relevant to a broad range of disciplines, including quantum optics ${ }^{1,2}$, highdensity data storage ${ }^{3}$, optical trapping ${ }^{4}$, astrophysics ${ }^{5,6}$, telecommunications ${ }^{7-13}$, high-resolution microscopy ${ }^{14}$, and optical interferometry for the detection of gravitational waves ${ }^{15,16}$. Photons entangled via OAM have also been used to demonstrate a violation of generalized Bell inequalities ${ }^{17}$ and for quantum cryptography ${ }^{18}$. Despite this broad range of applications, a method to dynamically discriminate a beam of a specific OAM state from other beams while retaining the initial state has not yet been reported in the literature.

The OAM of light is associated with the helical phase front of a propagating beam, just as spin angular

\footnotetext{
Correspondence: Jiao Lin (jiao.lin@unimelb.edu.au) or Shan Shan Kou (s.kou@latrobe.edu.au) or Xiao-Cong Yuan (xcyuan@szu.edu.cn) ${ }^{1}$ Nanophotonics Research Center, Shenzhen Key Laboratory of Micro-Scale Optical Information Technology, Shenzhen University, Shenzhen 518060, China ${ }^{2}$ Department of Chemistry and Physics, La Trobe Institute for Molecular Science (LIMS), La Trobe University, Victoria 3086, Australia

Full list of author information is available at the end of the article.

These authors contributed equally: Shibiao Wei, Stuart K. Earl
}

momentum is connected to the circular polarization of light. Laguerre-Gauss $(L G)$ laser modes were the first modes to be identified as carrying $\mathrm{OAM}^{19}$, although any beam with an azimuthal phase dependence proportional to $\exp (i l \theta)$ has $l \hbar$ units of OAM per photon, where the integer number $l$ is known as the topological charge and $\theta$ is the azimuthal coordinate. A beam of the OAM state $|l=0\rangle$ has a standard Gaussian intensity distribution, while a beam of any other OAM state has an intensity void (optical vortex) in its center owing to a phase singularity arising from its helical phase profile ${ }^{20}$.

A vortex beam of topological charge $+l$ can be converted into a Gaussian beam $(|l=0\rangle)$ by passing the beam through an optical element that has the opposite helical phase $\exp (-i l \theta)$; the resultant Gaussian beam can then be coupled into a single-mode optical fiber. All other copropagating OAM states will be unable to couple into the fiber because of the singularity at each of their centers, thus forming a simple mode-selection device ${ }^{21}$. A more intricate conformal transformation has been used to convert the helical phase structure of a vortex beam into a linear phase gradient using either spatial light modulators $(\mathrm{SLMs})^{22}$ or custom-designed elements ${ }^{23-25}$. The 
resultant linear spread of the beam profile is able to spatially disperse OAM states based on the topological charge. Spatial dispersion of OAM states was also recently reported using a plasmonic metasurface ${ }^{3}$ in which nanostructured grooves in a metallic film coupled the incident OAM states to surface plasmon polaritons on a metal surface and subsequently spatially routed them to nano-ring slits based on their topological charge. Other techniques, focusing at different focal planes ${ }^{26}$ and onchip sorting with plasmonics ${ }^{27}$, have also been reported. While spatially dispersing vortex beams through the conversion of the helical phase is an ingenious approach, it destroys the incident OAM states and is therefore more suitable for detecting the topological charge at the endpoint of an optical system. A more general sorting mechanism would be one that sends different OAM states to different output ports without altering the original states. A modified Mach-Zehnder interferometer consisting of Dove prisms and spiral phase plates has been used to construct a sorting device to select beams based on their OAM, $l$, and total angular momentum, $j=s+l$, where $s$ is the spin angular momentum ${ }^{1,2}$. However, this method still changes the incident OAM states because it uses spiral phase plates-however, the recovery of the original states is relatively easy. In theory, an interferometric method can sort a number of OAM states. However, sorting devices using this approach require $(n-1)$ interferometers with $(2 n-2)$ arms grouped at several stages to sort $n$ different OAM states, which means that the complexity and losses increase rapidly with the number of OAM states to be sorted. Furthermore, a unique combination of the configurations of all interferometer arms has to be found for each given set of OAM states. The configuration at each stage of this form of interferometer network may then also need to be completely modified with the inclusion of each new OAM state. In this work, we demonstrate dynamic sorting of OAM states by using cascaded tunable resonators. By changing the resonance status of a resonator, individual OAM states are separated.

\section{Results}

We propose a modular OAM sorting process (illustrated in Fig. 1a). In our process, each module must accept a number of input OAM states, output only one state, and divert all other states in an unaltered manner for subsequent processing. Therefore, within this modular architecture, the design of an OAM sorting system may be realized by simply cascading the modules, and the inclusion of any new OAM state can be achieved easily by connecting an additional module to the end of the chain. For the cascaded arrangement to work, it is important that each module rejects non-output OAM states with a very high efficiency so that the last output OAM state does not suffer from a significant intensity drop relative to the intensity of the first output. Mirrors are reflective

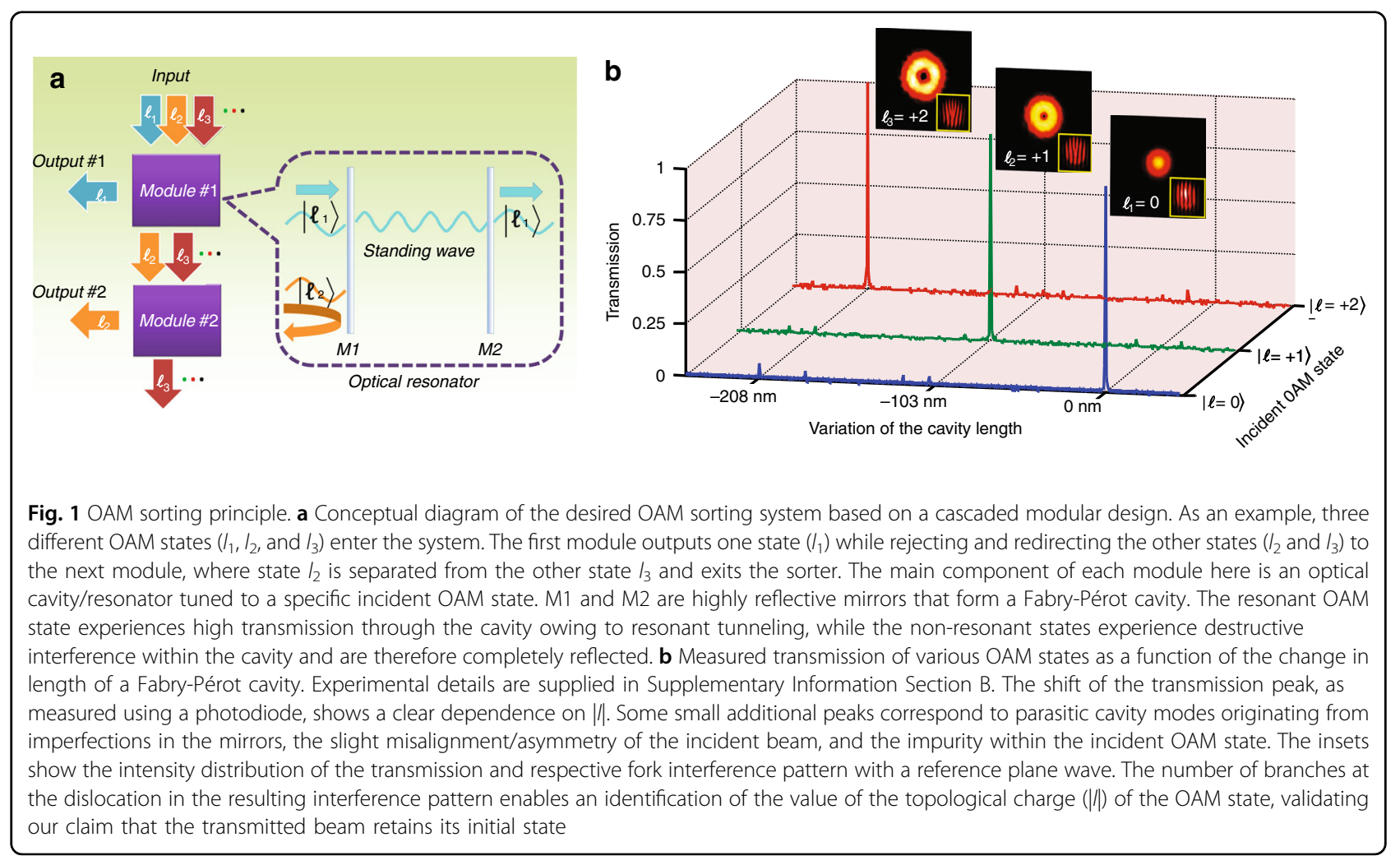


optical components that can reflect (reject) incident light with almost $100 \%$ efficiency $(99.9 \%$ efficiency is readily achieved by modern mirrors over the visible or infrared spectrum) while preserving most of the characteristics of the original light. However, a normal mirror cannot differentiate OAM states and acts as a barrier to all incident photons carrying different OAM states. Surprisingly, by adding another mirror (the second barrier shown in the inset of Fig. 1a), a particular OAM state can tunnel through the two barriers formed by the pair of mirrors in a fashion similar to a resonant tunneling diode. The space between the two mirrors can be considered to be an optical cavity that resonates with the selected OAM state, resulting in the tunneling of the state through the two mirrors. This configuration is, in fact, a Fabry-Pérot (FP) cavity $^{28}$, a ubiquitous, high-finesse optical resonator that is used in numerous applications ranging from large-scale interferometers $^{29}$ and resonant mode cleaning ${ }^{30}$ to astronomy $^{31}$ and spectroscopy ${ }^{32}$. The transmission of an FP cavity can approach $100 \%$ with an extremely high resolution $^{33}$, and the resonant properties can be manipulated by altering the cavity length, incident angle, and reflectivity of the mirrors ${ }^{34,35}$. Practically, most FP cavities are formed by two curved mirrors instead of parallel plane mirrors to reduce the beam walk-off and simplify the alignment of the incident beam. A propagating OAM state acquires a phase shift relative to a plane wave as it is focused by the curved mirrors. The accumulated phase shift varies with the OAM state and determines whether the state can form a standing wave in the cavity, i.e., at resonance. This phase shift is known as the Gouy phase shift and occurs as the beam propagates through the region around its focal point, which is $(z)=$ $\tan ^{-1}\left(z / z_{R}\right)\left(z_{R}\right.$ is the Raleigh range of the beam). This phase shift term only depends on the cavity parameters, and this additional phase shift enables a resonant cavity to sort vortex beams of different OAM states. A resonant mode of a cavity was defined by Kogelnik as a selfconsistent field configuration ${ }^{34}$; if a mode can be represented as a wave traveling back and forth between two mirrors, the beam parameters must by necessity be unchanged after one return trip around the cavity. This implies that in an optical cavity, resonance may only occur when the phase shift from one mirror to the other mirror is a multiple of $\pi$. An implicit relation between the cavity length and the topological charge of a resonant OAM state can be found (Methods) and expressed as

$$
D=\frac{\lambda}{2}\left[q+(|l|+1) \frac{\phi}{\pi}\right]
$$

where $D$ is the cavity length; $\phi=$ $\arccos \left( \pm \sqrt{\left(1-D / R_{1}\right)\left(1-D / R_{2}\right)}\right)$ is the accumulated Gouy phase shift experienced by the light traveling from one end of the cavity to the other, which is determined by the mirror curvatures $\left(R_{1}\right.$ and $\left.R_{2}\right) ; \lambda$ is the wavelength of light; and $q$ is an integer. Since the resonance is sensitive to even subwavelength movements (e.g., a few hundred nanometers), the transcendental equation can be approximated by an explicit relation between a small variation $\Delta$ in the cavity length and the resonant topological charge (Supplementary Information Section A):

$$
\Delta=\frac{\lambda}{2}\left[q+(|l|) \frac{\phi}{\pi}\right]
$$

Hence, one can resonantly match a specific OAM state to an FP cavity by tuning the cavity length, producing an OAM filter that allows the transmission of a selected state and the rejection of all others. The resonant filter then serves as the key component in the constituent module of an OAM sorting system.

A scanning FP cavity whose length can be actively tuned using a piezoelectric transducer was selected for the sorting experiment. A liquid-crystal-based spatial light modulator (SLM) was used to prepare various incident OAM states ${ }^{36}$.

Because an FP cavity with a high finesse is highly sensitive to wavelength instabilities and the broad spectrum of the OAM states, a frequency-stabilized $\mathrm{HeNe}$ laser with a wavelength of $632.8 \mathrm{~nm}$ is used. A frequency instability of the laser will lead to adrift of the resonant peaks. In an actual application, decreasing the finesse of the cavity will widen the resonant peak, increasing the tolerance to peak drift but limiting the number of OAM states that the system can distinguish. Therefore, there should be a trade-off between the performance of the laser beam and the finesse of the cavities in actual applications.

Figure $1 \mathrm{~b}$ clearly shows that a sharp transmission peak occurs at different cavity lengths for each OAM state. In other words, the FP cavity acts as a filter, transmitting a specific OAM state for every correctly selected cavity length. At resonance, we also verified that the transmitted vortex beam remains in its original OAM state. These experimental results are corroborated by numerical calculations performed using FINESSE ${ }^{37}$, an interferometer simulation package. The (absolute) cavity length used in these simulations was calculated using the change in the cavity length relative to the original resonated cavity length of a Gaussian beam using the $l$-dependent Gouy phase accumulated by a vortex beam within the FP cavity (see Supplementary Information Section C).

\section{Separation of co-propagating OAM states}

As an essential part of an OAM sorter, the FP cavity must be able to isolate a specific OAM state from a number of co-propagating states. Figure 2 shows that the FP cavity dynamically disperses the input beam, 


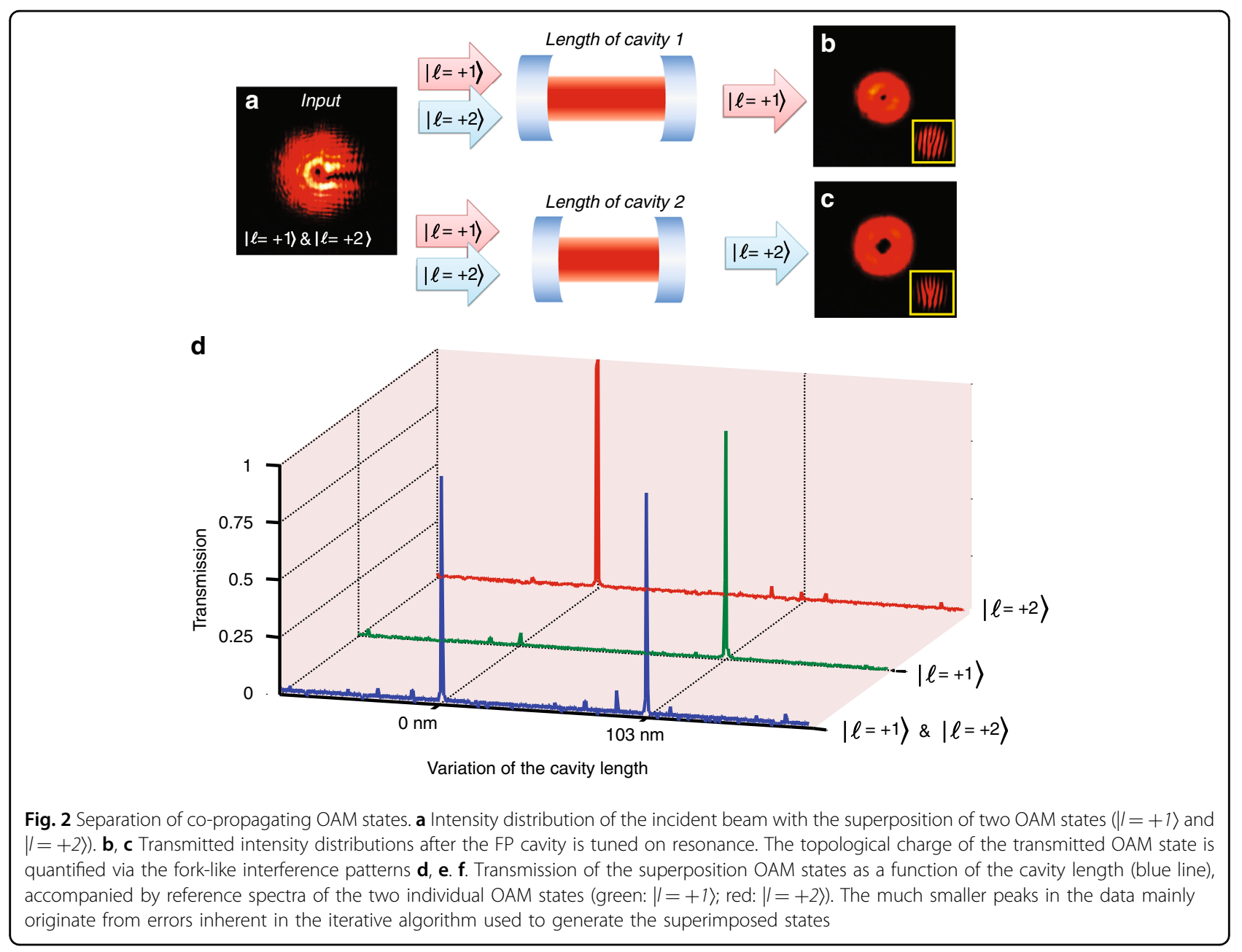

transmitting each constituent OAM state at different cavity lengths. To prepare a laser beam carrying two distinct OAM states $(|l=+1\rangle$ and $|l=+2\rangle)$ using a single SLM, an iterative algorithm was used to create the appropriate phase pattern ${ }^{38}$, which was then displayed on the SLM to convert an incident Gaussian beam into the superposition of two vortex states used here. By tuning the cavity length, different OAM states were selected. To intuitively detect the topological charges of the selected OAM states, the interference patterns between the OAM beam and a plane wave with a tilt incident angle were obtained, as shown in Fig. 2d, e. Two distinctive transmission peaks occur at the same cavity lengths as those of the reference beams carrying a single OAM state, as shown in Fig. 2f. The much smaller peaks in the data primarily originate from errors inherent in the iterative algorithm used to generate the superimposed states. The temperature variation and the displacement gaps between the increasing voltage and the decreasing voltage will influence the accuracy of the measurement of the cavity length. The interval between the states $(|l=+1\rangle$ and $|l=+2\rangle)$ was $103 \mathrm{~nm}$, which had a $2 \mathrm{~nm}$ shift compared with the value in Fig. 1.

\section{Modular design of an OAM sorter}

For the off-resonant OAM states, the FP cavity consists simply of two mirrors with very high reflectances. The high reflectivity, coupled with the fact that the cavity preserves all incident OAM states (for both transmission and reflection), enables the implementation of a modular design in which multiple cavities are cascaded, allowing simultaneous sorting of multiple OAM states. To demonstrate this implementation, a second FP cavity is added to construct an OAM sorter with two output ports, as shown in Fig. 3. An optical circulator ${ }^{37}$ is used to direct the reflected states from the first cavity to the second cavity. We show that it is possible to separate OAM states using cascaded cavities, enabling the simultaneous sorting/detection of multiple copropagating OAM states within a single beam. Furthermore, the path taken by a particular OAM state within the sorter can be manipulated in real time by altering the cavity lengths of the modules, permitting dynamic routing of an 


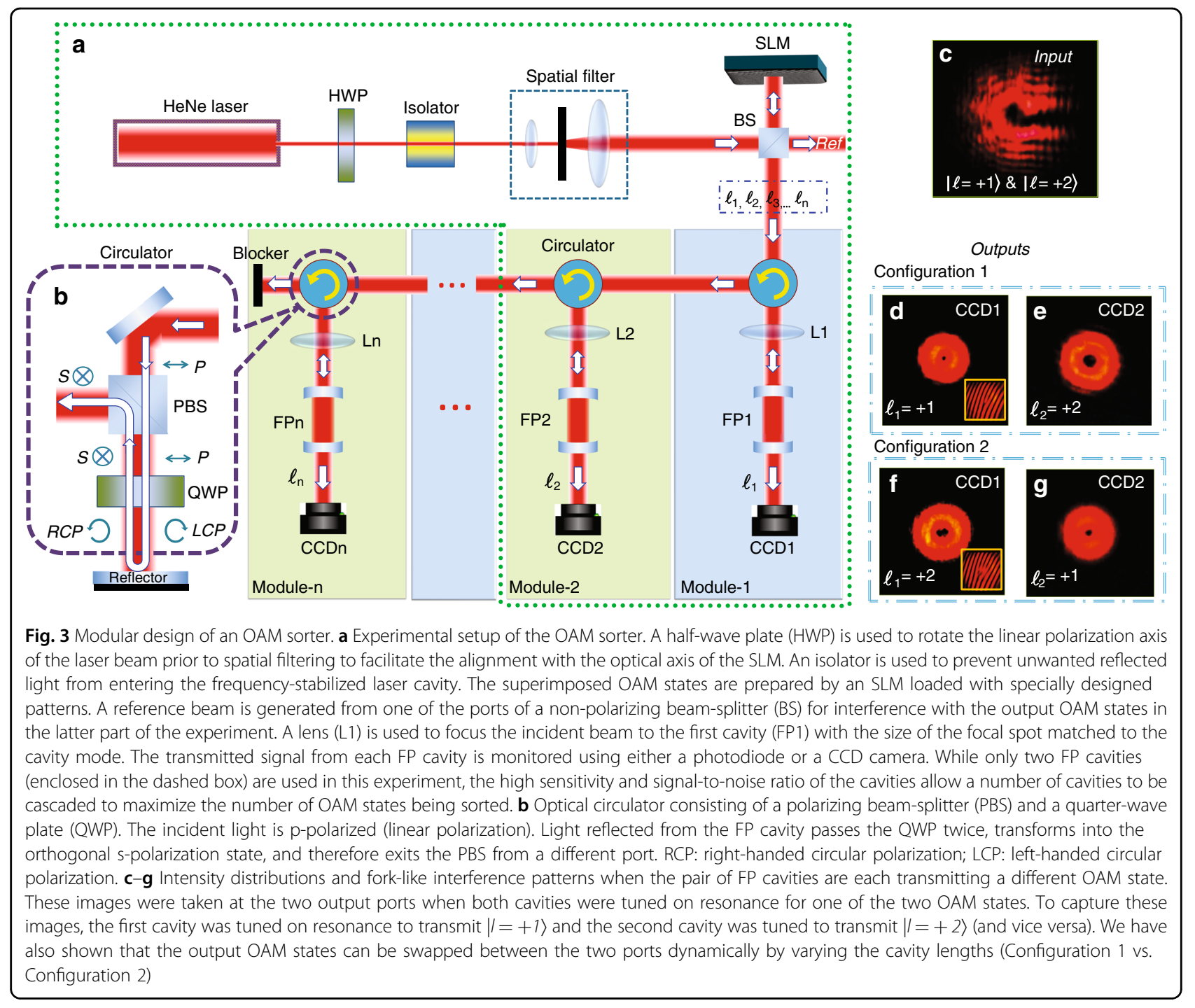

OAM state to any output port. Since the resonant cavity length only depends on the modulus of the topological charge, as indicated by Eq. 1, two OAM states with topological charges of opposite sign are degenerate and cannot be differentiated using a single FP cavity. One can choose to either use only positive (or negative) topological charges in the system or add spiral phase plates and a second FP cavity to the module to remove the degeneracy (Supplementary Information Section D).

\section{Simultaneous sorting of OAM and wavelength}

FP cavities are regularly used to differentiate finely spaced laser lines. This ability complements our approach to sorting OAM states by permitting simultaneous differentiation of optical signals based on both wavelength and the OAM state within the same device. Here we demonstrate simultaneous sorting of wavelengths and OAM states using a single FP cavity.
The frequency-stabilized $\mathrm{HeNe}$ laser used in the previous sections of this paper was replaced with another HeNe laser that is known to lase on three longitudinal modes (Melles Griot 05-LHR-151, with a mode spacing of $438 \mathrm{MHz}$, producing three laser lines with a separation of approximately $0.000584 \mathrm{~nm}$ ) centered at $632.816 \mathrm{~nm}$ in air. The remainder of the experimental setup remained unchanged, as shown in Fig. 1. The three laser lines, which would be indistinguishable using a diffraction grating, are clearly visible in Fig. 4 owing to the superior resolution of the FP cavity.

An input superposition of two OAM states $(|l=+1\rangle$ and $|l=+2\rangle)$ across the three wavelengths of the laser was directed through the FP cavity. The results of this experiment are shown in Fig. 4 (red line) with additional results from the single OAM state for reference. The six transmission peaks account for all possible combinations of the two OAM states and the three wavelengths. These 

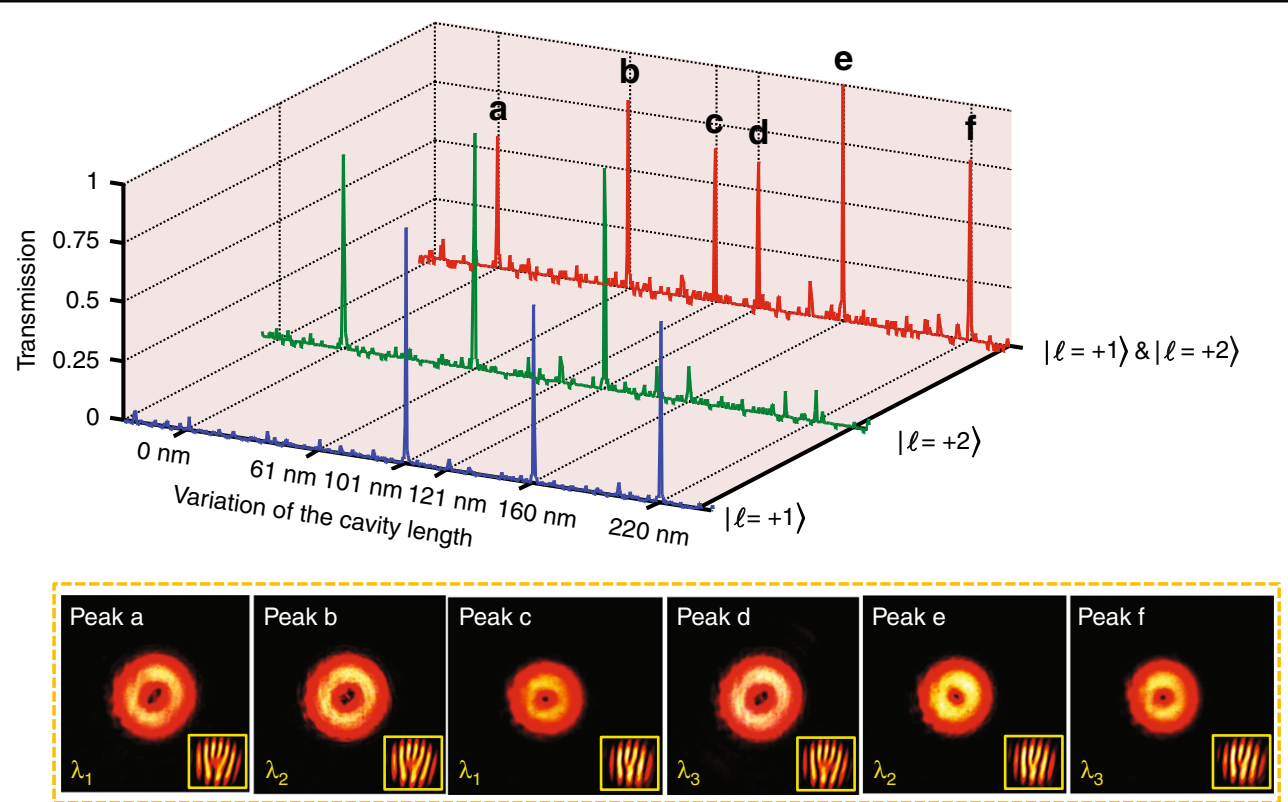

Fig. 4 Simultaneous sorting of OAM and wavelength. Simultaneous OAM and wavelength multiplexing/demultiplexing in an incident beam. The blue and green lines indicate the single OAM states generated from the same laser with three laser lines (the central wavelength is $632.816 \mathrm{~nm}$ in air, and the separation of each laser line is $0.000584 \mathrm{~nm}$ ), while the red line represents an incident beam with both OAM states at the three laser lines The peaks of the mixed OAM states match those of the individual OAM states. Insets: CCD images of each of the six resonant transmissions

results show that the OAM sorter can be used to separate wavelengths simultaneously without requiring additional components.

\section{Discussion}

In conclusion, we have demonstrated a reconfigurable OAM sorter that handles multiple OAM states simultaneously while preserving the original states. In addition, with this modular design, the system can be easily scaled with an increasing number of OAM states. The basic building element of this OAM sorter is an optical cavity; thus, the sorter inherits a number of merits from using a resonance-based approach, such as high selectivity and high efficiency. The cross-talk between the various OAM states is suppressed because of the resonant nature of this approach. Furthermore, by harnessing the principle of Gouy phase accumulation, the sorter is sensitive not only to the values of the azimuthal mode index $l$ but also to the radial mode index $p$. Compared with an interferometer that was previously proposed for sorting the radial modes of $L G$ beams ${ }^{39}$, the sorter proposed here is more compact, which is important when considering actual applications. The transmission of the resonant OAM states is experimentally measured to be approximately $92 \%$ of the maximum efficiency (21.7\%) of the particular off-the-shelf FP cavity used in the experiment. While FP cavities can theoretically be $100 \%$ efficient, in practice, they are limited by the surface roughness of the mirror coatings/surfaces, alignment errors, and other imperfections. For a high- finesse cavity, such as those used in cavity quantum electrodynamics (QED) experiments, scattering and absorption losses from the mirrors are the major sources of loss ${ }^{40}$. Moving from the visible region $(\sim 632.8 \mathrm{~nm})$ to the infrared region to minimize surface scattering and material absorption losses is the simplest way to improve the efficiency. An optimization of the FP cavity and the use of dielectric mirrors would also increase the efficiency. For example, the transmission efficiency could be significantly improved with a high-efficiency cavity and a longer working wavelength; for example, over 99\% transmission efficiency has been reported experimentally using mirrors with a higher reflectance in the cavity and by moving to longer wavelengths such as $1064 \mathrm{~nm}$ (Supplementary Information Section $E)^{41}$ ). Furthermore, because an FP cavity with a high finesse is highly sensitive to wavelength instabilities and the broad spectrum of the OAM states, a frequency-stabilized $\mathrm{HeNe}$ laser with a wavelength of $632.8 \mathrm{~nm}$ is used in the experiment. A frequency instability of the laser will lead to a drift of the resonant peaks. In an actual application, decreasing the finesse of the cavity will widen the resonant peak, thus increasing the tolerance to peak drift but also limiting the number of OAM states that the system can distinguish. This trade-off between the performance of the laser beam and the finesse of the cavities will therefore need to be considered in light of specific real-world applications. Furthermore, we have reported an OAM healing effect by using an FP cavity ${ }^{42}$. An obstructed OAM beam can be 


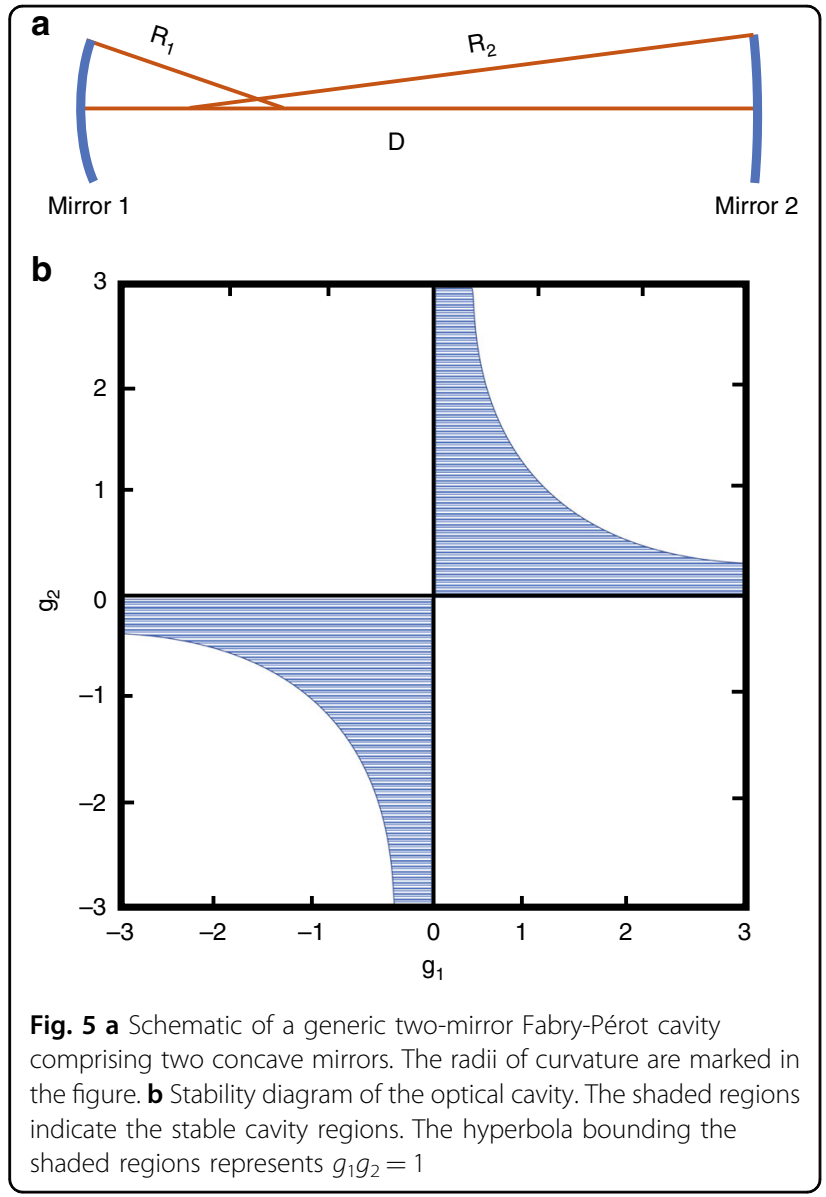

healed when passing through an FP cavity, which will greatly increase the stability of the whole OAM application system.

In conjunction with wavelength and polarization, modedivision multiplexing (MDM) using optical OAM states has been investigated because of the infinite number of orthogonal states potentially available for increasing the transmission capacity of a network. OAM-enabled optical networks have already demonstrated capacities greater than 1 Terabit per second ${ }^{7,8,13}$. While increases in the datacarrying capacity of single-mode fibers have outweighed the demand over the course of the previous three decades, our ever-expanding demand for more data is predicted to exceed the current capacities within a decade ${ }^{43}$. The dynamic nature of the OAM sorter offers a simple avenue for constructing reconfigurable optical networks to address this shortfall from another perspective. The mature nature of optical cavity technology, the high quality of available materials, and the ability to reduce the cavity dimensions down to on-chip and in-fiber cavities all indicate that this approach could have a wide-reaching impact. Our OAM sorting technique may also be of interest to imaging, as hyperspectral imaging camera arrays now integrate FP cavities on a single-pixel level ${ }^{44}$, and quantum optics researchers may find interesting implications in these findings for cavity QED experiments ${ }^{45,46}$.

\section{Materials and methods}

The mechanism behind the OAM-sorting ability of a Fabry-Pérot (FP) cavity is the Gouy phase shift that a resonant beam accumulates within the cavity. This phenomenon, in which a propagating wave acquires a phase shift relative to a (theoretical) plane wave as it is focused by an optical system, was first observed in $1890^{47}$.

Figure 5a shows a schematic of a generic two-mirror optical cavity comprising concave mirrors with different radii of curvature. The stability of such a cavity can be calculated by ray transfer analysis ${ }^{34}$. The result of this analysis commonly introduces the $g$ factors of a cavity, defined as

$$
g_{1,2}=1-D / R_{1,2}
$$

where $R_{1,2}$ is the radius of curvature of the specified mirror and $D$ is the mirror separation, such that a cavity is stable provided that

$$
0 \leq g_{1} g_{2} \leq 1
$$

A stable cavity is one in which a paraxial ray injected into the resonator does not escape but rather remains confined near the longitudinal axis of the cavity. In contrast, an unstable cavity will allow light, even on-axis light, to eventually escape. Fig. 5b illustrates the combinations of the $g$ factors that result in a stable cavity in the shaded regions. The commercial scanning FP cavity used in our experiment was originally confocal $\left(D=R_{1}=R_{2}\right)$, corresponding to the origin $(0,0)$ in the above diagram. The advantage of a confocal FP cavity is that all longitudinal and transverse modes occur at the same cavity length. Since this experiment relied on a cavity to disperse the input spatial modes, the cavity length was extended $\left(R_{1}=\right.$ $R_{2}=R<D<2 R$ ) so that the cavity was no longer the case. The resultant cavity resides in the lower-left quadrant of the stability diagram owing to the convention of concave mirrors having a positive radius of curvature in this context.

To study the detailed field distributions within the FP cavity, we chose Laguerre-Gauss (LG) modes, a family of solutions to the paraxial wave equation in cylindrical coordinates, which, as discussed, were previously shown 
to carry $\mathrm{OAM}^{19}$. The general solutions are of the form

$$
\begin{aligned}
u_{p l}(r, \theta, z)= & \frac{C}{\sqrt{1+z^{2} / z_{R}^{2}}}\left(\frac{r \sqrt{2}}{w(z)}\right)^{l} L_{p}^{l}\left(\frac{2 r^{2}}{w(z)^{2}}\right) \\
& \times \exp \left(\frac{-r^{2}}{w(z)^{2}}\right) \exp \left(\frac{-i k r^{2} z}{2\left(z^{2}+z_{R}^{2}\right)}\right) \\
& \times \exp (-i l \theta) \exp (i(2 p+|l|+1) \psi(z))
\end{aligned}
$$

In this equation, $u_{p l}$ is the eigensolution to the paraxial wave equation; $C$ is a normalization constant; $L_{p}{ }^{l}$ is the Laguerre-Gauss polynomial of order $(p, l) ; p$ and $l$ are the radial and azimuthal mode indices, respectively; $w(z)$ is the standard definition of the beam waist; $r, \theta$, and $z$ are the radial, azimuthal and longitudinal coordinates, respectively; and $z_{R}$ is the Raleigh range of the beam, defined as

$$
z_{R}=\frac{\pi w_{0}^{2}}{\lambda}
$$

Here, $w_{0}$ is the standard definition of the beam waist. The Raleigh range is the distance the beam needs to propagate from the waist for the width of the beam to increase by $\sqrt{2}$, which corresponds to an on-axis intensity of half the peak intensity at that point.

The term $\psi(z)=\tan ^{-1}\left(z / z_{R}\right)$ represents the Gouy phase shift as the beam propagates through the region around its focal point. The above equation shows that for higher-order $L G$ modes, there is an additional Gouy phase shift relative to a Gaussian beam owing to the additional transverse structure of the beam. This additional phase shift enables a resonant cavity to sort vortex beams of different OAM states.

A resonant mode of a cavity was defined by Kogelnik as a self-consistent field configuration ${ }^{34}$; if a mode can be represented as a wave traveling back and forth between two mirrors, the beam parameters must by necessity be unchanged after one return trip around the cavity. This implies that in an optical cavity, resonance may only occur when the phase shift from one mirror to the other mirror is a multiple of $\pi$.

The total phase accumulated by an $L G$ beam traveling from one side of a stable optical cavity to the other side (i.e., mirror 1 at $z_{1}$ and mirror 2 at $z_{2}$ ) can be written as

$$
\emptyset\left(z_{2}-z_{1}\right)=k D-(2 p+|l|+1)\left[\psi\left(z_{2}\right)-\psi\left(z_{1}\right)\right]
$$

The first term on the right-hand side represents the phase advance caused by light with a propagation constant $k$ traveling a distance $D$, while the second term represents the accumulated Gouy phase within the cavity. This second term was shown by Siegman to depend only on the cavity parameters ${ }^{33}$, i.e.,

$$
\psi\left(z_{2}\right)-\psi\left(z_{1}\right)=\phi=\arccos \left( \pm \sqrt{g_{1} g_{2}}\right)
$$

where the $g$ factors are as defined previously. The choice of sign in the definition of the Gouy phase depends on the position of the cavity in the stability diagram (Fig. 5b). The "+" sign applies to the upper-right quadrant, while the “-” sign applies to the lower-left quadrant.

Based on the previous equation and the knowledge that the condition for a standing wave is that the total round trip (total distance traveled of $2 D$ ) phase must be an integer multiple of $2 \pi$, a mode must satisfy the following equation to be resonant with the cavity:

$$
\frac{\omega 2 D}{c}-2(2 p+|l|+1) \phi=2 q \pi
$$

where $q$ is an integer. Here, the substitution of $k=\frac{\omega}{c}$ was used to facilitate the derivation of the resultant resonance frequencies of the longitudinal-plus-transverse modes of the cavity, i.e.,

$$
\omega=\omega_{q p l}=\frac{\pi c}{D}\left(q+\frac{(2 p+|l|+1)}{\pi} \phi\right)
$$

where the factors of 2 have been canceled. This equation tells us that for a cavity with a fixed length $D$, different $L G$ modes will resonate with the fixed cavity at slightly different frequencies because of the influence of the Gouy phase.

For a dynamic cavity such as the scanning FP cavity used in this experiment, it is possible to rearrange the previous equation, as $D$ is variable while the frequency is fixed. The result is that for an $L G_{p}^{l}$ mode of wavelength $\lambda$ within a cavity, the resonant cavity length, $D$, is

$$
D=\frac{\lambda}{2}\left\{q+(2 p+|l|+1) \frac{\phi}{\pi}\right\}
$$

Here, $\lambda=2 \pi c / \omega$ was used to simplify the resulting equation. This equation shows that owing to the accumulated Gouy phase shift experienced by a beam traveling from one mirror to the other mirror, the resonant length of the cavity depends on the values of the azimuthal mode index (OAM topological charge) and the radial mode index. Since we are interested in the sorting of OAM (the azimuthal mode index $l$ ), the radial mode index $p$ is irrelevant in this case and thus is set to zero to further simplify the equation:

$$
D=\frac{\lambda}{2}\left\{q+(|l|+1) \frac{\phi}{\pi}\right\}
$$

This dependence is the mechanism that enables the FP cavity to differentiate vortex beams of different OAM states. As the cavity length is varied, different OAM states 
resonate within the cavity (and are transmitted), while all other states experience destructive interference (and are therefore reflected).

\section{Acknowledgements}

This work is partially supported the National Natural Science Foundation of China under grant Nos. U1701661/61935013/61427819/11604219/61675136; The National Key R\&D Program of China under grant No. 2018YFB1801801; the Leading Talents Program of Guangdong Province under grant No. 00201505; the Natural Science Foundation of Guangdong Province under grant No. 2016A030312010; and the Shenzhen Science and Technology Innovation Commission under grant No. KQJSCX20170727100838364/ KQTD2015071016560101/KQTD20180412181324255. S.W. acknowledges the support of the Endeavour Australia Cheung Kong Research Fellowship funded by the Department of Education and Training, Australia. The authors would like to thank Professor Baohua Jia (Swinburne University of Technology) and Professor Ann Roberts (The University of Melbourne) for the loan of the equipment.

\section{Author details}

${ }^{1}$ Nanophotonics Research Center, Shenzhen Key Laboratory of Micro-Scale Optical Information Technology, Shenzhen University, Shenzhen 518060, China. ${ }^{2}$ Department of Chemistry and Physics, La Trobe Institute for Molecular Science (LIMS), La Trobe University, Victoria 3086, Australia. ${ }^{3}$ School of Engineering, RMIT University, Melbourne, Victoria 3001, Australia. ${ }^{4}$ School of Physics, The University of Melbourne, Tin Alley, Melbourne, Victoria 3010, Australia

\section{Authors contributions}

J.L. conceived the concept. J.L., S.K.E. and S.W. designed the experiment. S.W. and S.K.E. performed the experiment. S.W., S.K.E., S.S.K. and J.L. acquired, analysed and interpreted the data. S.K.E. drafted the manuscript with inputs from all other authors. J.L., S.S.K. and X.Y. supervised the project, revised the manuscript with a critical eye, and contributed significantly to its intellectual content.

\section{Conflict of interest}

The authors declare that they have no conflict of interest.

Supplementary information is available for this paper at https://doi.org/ 10.1038/s41377-020-0243-x.

Received: 24 August 2019 Revised: 23 December 2019 Accepted: 25 December 2019

Published online: 28 January 2020

\section{References}

1. Leach, J. et al. Interferometric methods to measure orbital and spin, or the total angular momentum of a single photon. Phys. Rev. Lett. 92, 013601 (2004).

2. Leach, J. et al. Measuring the orbital angular momentum of a single photon. Phys. Rev. Lett. 88, 257901 (2002).

3. Ren, H. R. et al. On-chip noninterference angular momentum multiplexing of broadband light. Science 352, 805-809 (2016).

4. He, H. et al. Direct observation of transfer of angular momentum to absorptive particles from a laser beam with a phase singularity. Phys. Rev. Lett. 75, 826-829 (1995)

5. Lavery, M. P. et al. Detection of a spinning object using light's orbital angular momentum. Science 341, 537-540 (2013).

6. Berkhout, G. C. \& Beijersbergen, M. W. Method for probing the orbital angular momentum of optical vortices in electromagnetic waves from astronomical objects. Phys. Rev. Lett. 101, 100801 (2008).

7. Bozinovic, N. et al. Terabit-scale orbital angular momentum mode division multiplexing in fibers. Science 340, 1545-1548 (2013).

8. Lei, T. et al. Massive individual orbital angular momentum channels for multiplexing enabled by Dammann gratings. Light Sci. Appl. 4, e257 (2015).
9. Yan, Y. et al. High-capacity millimetre-wave communications with orbital angular momentum multiplexing. Nat. Commun. 5, 4876 (2014).

10. Gibson, $G$. et al. Free-space information transfer using light beams carrying orbital angular momentum. Opt. Express 12, 5448-5456 (2004).

11. Lin, J. et al. Multiplexing free-space optical signals using superimposed collinear orbital angular momentum states. Appl. Opt. 46, 4680-4685 (2007).

12. Fu, S. Y. et al. Demonstration of free-space one-to-many multicasting link from orbital angular momentum encoding. Opt. Lett. 44, 4753-4756 (2019).

13. Wang, J. et al. Terabit free-space data transmission employing orbital angular momentum multiplexing. Nat. Photonics 6, 488-496 (2012).

14. Ritsch-Marte, M. Orbital angular momentum light in microscopy. Philos. Trans. A Math. Phys. Eng. Sci. 375, 20150437 (2017).

15. Bondarescu, M. \& Thorne, K. S. New family of light beams and mirror shapes for future LIGO interferometers. Phys. Rev. D 74, 082003 (2006).

16. Mours, B., Tournefier, E. \& Vinet, J. Y. Thermal noise reduction in interferometric gravitational wave antennas: using high order TEM modes. Classical Quant. Grav. 23, 5777-5784 (2006).

17. Dada, A. C. et al. Experimental high-dimensional two-photon entanglement and violations of generalized Bell inequalities. Nat. Phys. 7, 677-680 (2011).

18. Gröblacher, S. et al. Experimental quantum cryptography with qutrits. New J. Phys. 8, 75 (2006).

19. Allen, L. et al. Orbital angular momentum of light and the transformation of Laguerre-Gaussian laser modes. Phys. Rev. A 45, 8185-8189 (1992).

20. Yao, A. M. \& Padgett, M. J. Orbital angular momentum: origins, behavior and applications. Adv. Opt. Photonics 3, 161-204 (2011).

21. Mair, A. et al. Entanglement of the orbital angular momentum states of photons. Nature 412, 313-316 (2001).

22. Berkhout, G. C. G. et al. Efficient sorting of orbital angular momentum states of light. Phys. Rev. Lett. 105, 153601 (2010)

23. Lavery, M. P. J. et al. Refractive elements for the measurement of the orbital angular momentum of a single photon. Opt. Express 20, 2110-2115 (2012).

24. Ruffato, G. et al. Test of mode-division multiplexing and demultiplexing in freespace with diffractive transformation optics. Opt. Express 25, 7859-7868 (2017)

25. Ruffato, G. et al. Total angular momentum sorting in the telecom infrared with silicon Pancharatnam-Berry transformation optics. Opt. Express 27, 15750-15764 (2019).

26. Mei, S. T. et al. Flat helical nanosieves. Adv. Funct. Mater. 26, 5255-5262 (2016).

27. Mei, S. T. et al. On-chip discrimination of orbital angular momentum of light with plasmonic nanoslits. Nanoscale 8, 2227-2233 (2016).

28. Fabry, C. \& Perot, A. Theorie et applications d'une nouvelle methode de spectroscopie interferentielle. Ann. Chim. Phys. 16, 115 (1899).

29. Abramovici, A. et al. LIGO: the laser interferometer gravitational-wave observatory. Science 256, 325-333 (1992).

30. Rüdiger, A. et al. A mode selector to suppress fluctuations in laser beam geometry. Opt. Acta Int. J. Opt. 28, 641-658 (1981).

31. De Graauw, T. et al. Observing with the ISO short-wavelength spectrometer. Astron. Astrophys. 315, L49-L54 (1996).

32. Jacquinot, P. New developments in interference spectroscopy. Rep. Prog. Phys. 23, 267-312 (1960).

33. Siegman, A. E. Lasers. (Mill Valley: University Science Books, 1986).

34. Kogelnik, H. \& Li, T. Laser beams and resonators. Proc. IEEE 54, 1312-1329 (1966).

35. Pedrotti, F. L., Pedrotti, L. M. \& Pedrotti, L. S. Introduction to Optics. 3rd edn. (Upper Saddle River, NJ.: Pearson Prentice Hall, 2007), 17-25.

36. Andrews, D. L. Structured Light and Its Applications: An Introduction to Phasestructured Beams and Nanoscale Optical Forces. (Academic Press, Amsterdam, The Netherlands, 2008).

37. Freise, A., Brown, D. \& Bond, C. Finesse, frequency domain INterferomEter simulation softwarE. arXiv preprint arXiv:1306.2973 (2013).

38. Lin, J. et al. Collinear superposition of multiple helical beams generated by a single azimuthally modulated phase-only element. Opt. Lett. 30, 3266-3268 (2005).

39. Gu, X. M. et al. Gouy phase radial mode sorter for light: concepts and experiments. Phys. Rev. Lett. 120, 103601 (2018).

40. Hood, C. J., Kimble, H. J. \& Ye, J. Characterization of high-finesse mirrors: loss, phase shifts, and mode structure in an optical cavity. Phys. Rev. A 64, 033804 (2001).

41. Sekiguchi, H. et al. Ultralow-loss mirror of the parts-in-106 level at $1064 \mathrm{~nm}$. Opt. Lett. 20, 530-532 (1995).

42. Wei, S. B. et al. Demonstration of orbital angular momentum channel healing using a Fabry-Pérot cavity. Opto-Electron. Adv. 1, 180006 (2018). 
43. Essiambre, R. J. \& Tkach, R. W. Capacity trends and limits of optical communication networks. Proc. IEEE 100, 1035-1055 (2012).

44. Agrawal, P. et al. Characterization of VNIR hyperspectral sensors with monolithically integrated optical filters. Proceedings of 2016 IS\&T International Symposium on Electronic Imaging 2016 Image Sensors and Imaging Systems. San Francisco, California, USA: Society for Imaging Science and Technology, 2016.
45. Hood, C. J. et al. Real-time cavity QED with single atoms. Phys. Rev. Lett. 80 4157-4160 (1998).

46. Hood, C. J. et al. The atom-cavity microscope: Single atoms bound in orbit by single photons. Science 287, 1447-1453 (2000).

47. Gouy, L. G. Surune Propriété Nouvelle Des Ondes Lumineuses (Gauthier-Villars, Paris, 1890). 